\title{
PLAN FOR BREEDING, MAINTENANCE AND PRODUCING THE NUCLEOLUS (BREEDER'S SEED) OF GIZA 87 EGYPTIAN COTTON VARIETY
}

\author{
AL-AMEER, M. A. \\ Cotton Research Institute, ARC, Giza, Egypt \\ (Manuscript received 3 September 2014)
}

\begin{abstract}
The breeding program of Giza 87 was conducted at Sakha Agricultural Research Station, Kafr El-Sheikh district during 2010 2013 growing seasons. In 2010 season sixty type plants were selected from the breeding field of Giza 87 variety and furnished sixty progenies (increase $A$ ) in 2011. From the later, seventeen families were selected to descend increase B in 2012. According to the statistical analysis of yield trial which included the seventeen families and comparisons of the latest lines of Giza 87, six elite families were selected and the seeds were carefully massed together to form the nucleolus (breeder's seed) in 2013 season. The results obtained indicated that, the pure line method in the sense of pedigree selection for renewing annually Giza 87 breeder's seed could produce high genetically pure seeds and in the same time, prevent genetic contamination. Meanwhile, the selection technique for producing breeder's seed of Giza 87 variety was valid and proved to be effective in holding this variety according to the standard type of Giza 87.
\end{abstract}

\section{INTRODUCTION}

Egyptian cotton ( $\boldsymbol{G}$. barbadense L.) is considered the best quality among fiber crop in the world cottons. Supplying cotton seeds to farmers involves three separate activities; 1- Variety maintenance effort through breeding annually a new nucleolus carried out by the Cotton Varietal Maintainance Research Section of the Cotton Research Institute (C.R.I.), 2- Seed increase administered by the Central Administration For Seed Production (C.A.S.P.) and 3- Seed certification administered by the Central Administration For Seed Certification (C.A.S.C.). Lewis (1970) indicated that varietal maintenance of the Egyptian cotton varieties played an important role in the breeding programs with the fact that high quality properties are the principal merit of Egyptian cotton, will deteriorate unless an effort is made to maintain it. In Egypt, after new cotton variety was developed by the breeders (Cotton Breeding Research Department subjected to Cotton Research Institute (C.R.I.), it should be subjected to scientific system for producing the new varieties. The scheme of breeding based on pure line theory using pedigree selection method for producing the breeder's seed of 
the new cotton cultivars. In commercial use; Cotton Varietal Maintainance Research Department is responsible of maintaining and renewing breeder's seed of commercial varieties. Maintenance of the Egyptian cotton varieties have been reported by many workers, Ware (1959), Turner (1963) Walker (1964) and Riggs (1967) studied a model bulk system designed to stabilize a variety. They concluded that this system could be considered as a good maintenance procedure for a variety already released. Al-Didi (1974) stated that it was advantageous to mass the seed of chosen progenies in which the seed mixture may respond differently to environmental variation. He added that, if genotype $x$ environment effects were significant, mixture of seeds might show less fluctuation in yield and quality than individual progenies. Also, El-Akkad et. al. (1982), El-Kilany and Youssef (1985), Younis et. al. (1993), Abo-Arab et. al. (1995), Lasheen (1997), El-Disouqi (2001), Nagib and Hemida (2001), Abd Al-Zaher (2004) and Mohamed (2013) stated that the pure seed and production of long and extra long staple varieties, using the pedigree selection method is necessary to produce, renew and maintain the breeder's seed of the cotton cultivars in commercial use. The present method of maintaining Egyptian cotton varieties is the pedigree method based on massing selfed seeds of several type families, according to their performance in evaluation with the latest nuclei. Therefore, the main objective of the present study is to follow the steps of renewing and maintaining the breeder's seed of Giza 87.

\section{MATERIALS AND METHODS}

Giza 87 cotton variety, cultivated in the Delta region, is classified extra long staple, with a staple length $(36-37 \mathrm{~mm}$.). This variety was derived by the pedigree selection method from the hybridization between Giza 45 and Giza 77 A Egyptian cotton varieties.

The base population used in the present study is the individual elite plants selected through field evaluation and laboratory determinations from breeding plot of 2009 season.

At harvest each individual plant in the breeding plot was picked separately. The plants were screened for testing yield and its components characteristics (boll weight, seed index and lint percentage) as well as fiber properties (fiber length, fineness (micronaire reading) and strength. Sixty plants representing the type of Giza 87 variety were selected, in 2010 season to furnish the increase lines A in 2011 season. 
In 2011 season, the selfed seeds of the progenies of the 60 selected type plants were grown in number of rows as the amount of seed allowed conveniently named increase lines $A$, as well as the natural pollinated seeds of the same 60 selected type plants were grown in adjacent progeny three rows to be increased for using it in yield trial in the next year. Accordingly the field and laboratory tests of phenotypic yield and its components and fiber properties, 17 families were selected from increase $A$.

In 2012 season, the selfed seeds of the 17 families were grown in increase B. A yield trial included the seventeen selected families, as well as the three latest strains of Giza 87 namely G. 87 / 2010, G. 87 / 2011 and G. 87 / 2012 nuclei were used as controls. This yield trial was conducted at Sakha Agricultural Research Station. The design of yield trial was a randomized complete block design with four replications. The twenty entries were evaluated for yield and its components and fiber properties i.e. fiber length, fineness (micronaire reading) and strength. 


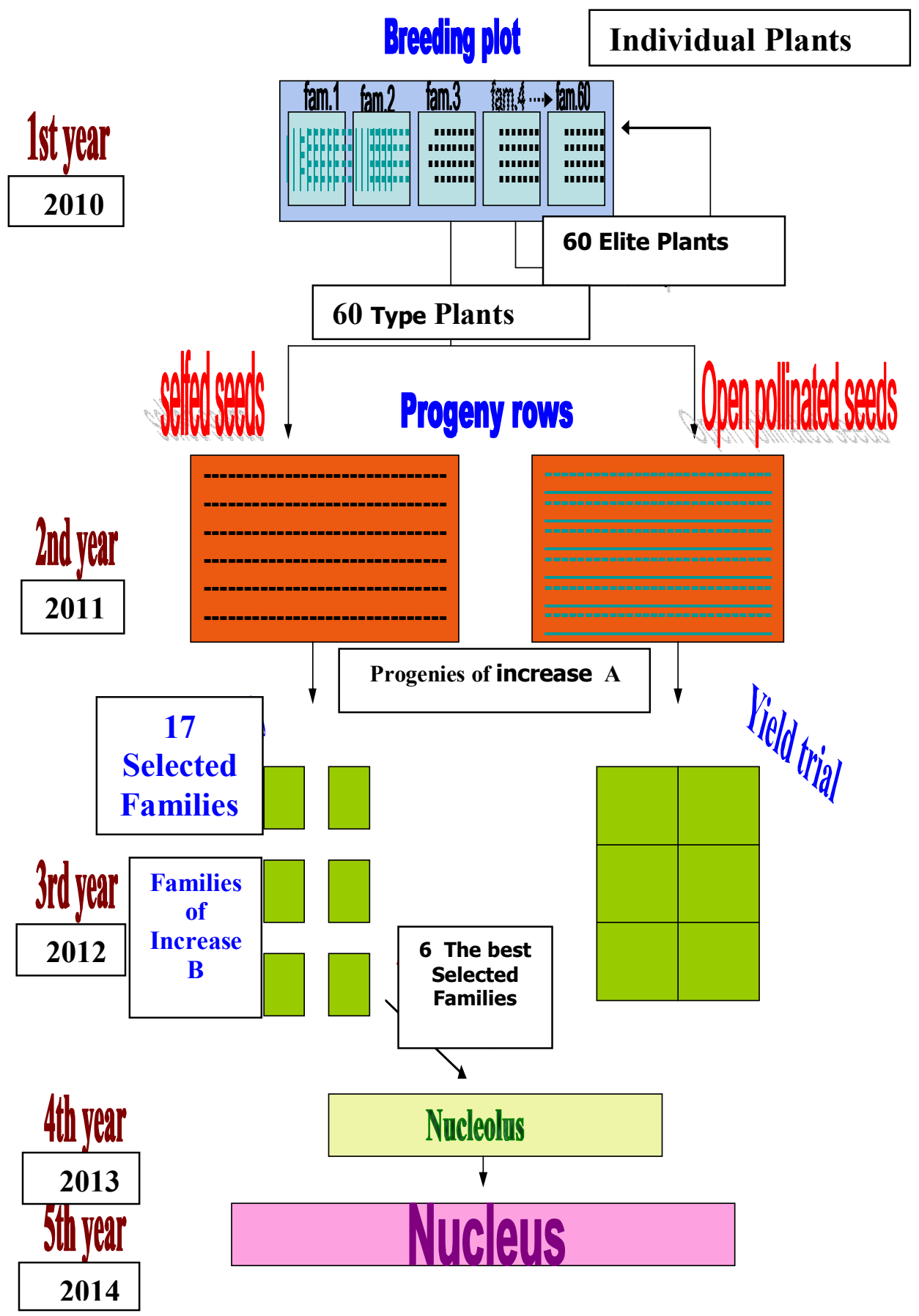

Figure 1. Maintaining System of Egyptian Cotton Varieties, Abdel-Al (1976)

The system used by the Cotton Maintenance Section, Cotton Research Institute, to maintain the Egyptian cotton varieties was described by Al-Didi (1974) and Abdel-Al (1976).This system was illustrated by Abdel-Al (1976) in Figure 1. 
In 2013 season, the best 6 families representing the type of Giza 87 variety were selected from increase $B$ and their selfed seeds were carefully massed together to form the new nucleolus (breeder's seed) of that variety and propagated in 2013 under the name of season (G. 87 nucleolus / 2013 ) in about 15 feddans at Sakha Farm.

Data were recorded in this research on the following traits:

Yield components characters are:

1- Seed cotton yield (S.C.Y.) in K. /F.

2- Lint cotton yield (L.C.Y.) in K. /F.

3- Boll weight (B.W.) in gm.

4- Lint percentage (L.P. \%)

5- Seed index (S.I.) in gm.

6- Lint index (L.I.) in gm.

Fiber properties traits are:

1- Fiber length (F.L.) Fiber length $2.5 \%$ in m.m.

2- Fiber fineness (F.F.) Micronaire reading.

3- Fiber strength (F.S.) Pressley index, Strength g/tex (ST.g/tex) and Yarn strength (Yarn stern.).

4- Uniformity ratio (U.R. \%).

5- Elongation (Elon.).

6- Yellowness $(+b)$

7- Brightness (RD \%).

Analysis of variance was conducted for all the studied characters in the yield trial and tested for significance by " $F$ " test.

Mean of selected families, mean of comparisons, standard error and coefficient of variability (C.V. \%) was executed for all characters.

\section{RESULTS AND DISCUSSION}

Means of agronomic characters and fiber properties for the selected 60 type plants of Giza 87 variety in 2010 seasons, are shown in Table 1, data indicated that no substantial differences for all studied characters were found. Whereas, coefficients of variability were low in magnitude for most studied characters except for boll weight in gram, lint index in gram, and fiber fineness (micronaire reading). This could be due to environmental effects on these traits. These results were in agreement with those obtained by Abo-Arab et. al. (1995), El-Disouqi (2001), Abd Al-Zaher (2004) and Mohamed (2013).

Also, the results showed that the lower values of coefficients of variability (C.V. $\%$ ) indicate that there is a lot of homogeneity within and between the selected type plants. These results indicated that there is a relationship between the selected 
characters in the different generations and it was constant, also there was no changes and no deviations in behaviour of the studied characters from generation to another because of an independent culling selection method was applied according to standard type of Giza 87 variety.

Table 1. Means of agronomic characters and fiber properties for the 60 selected type plants of Giza 87 from 2010 season and will form the increase A in 2011 growing season.

\begin{tabular}{|c|c|c|c|c|c|c|c|c|c|}
\hline No. & $\begin{array}{c}\text { Type Plants } \\
\text { No. }\end{array}$ & $\begin{array}{c}\text { Boll } \\
\text { Weight } \\
\text { gm. }\end{array}$ & $\begin{array}{c}\text { Lint } \\
\text { Percent } \\
\%\end{array}$ & $\begin{array}{l}\text { Seed } \\
\text { Index } \\
\text { gm. }\end{array}$ & $\begin{array}{l}\text { Lint } \\
\text { Index } \\
\text { gm. }\end{array}$ & $\begin{array}{c}\text { Fiber } \\
\text { Length } \\
2.5 \% \\
\mathrm{~mm}\end{array}$ & U.R.\% & F.F. & $\begin{array}{c}\text { Pressley } \\
\text { index }\end{array}$ \\
\hline 1 & $1 / 2010-5$ & r.q & r... & $1 . .1$ & 纟.० & ro.1 & ᄉ9.乏 & r.r & $11 . \mathrm{V}$ \\
\hline 2 & $1 / 2010-38$ & r.A & T..0 & 9.0 & $\varepsilon . \varepsilon$ & r..0 & 19.1 & r.乏 & $11 .$. \\
\hline 3 & $1 / r+1 .-r q$ & r.A & M. & 9.0 & 纟.r & ro.0 & $9 \ldots$ & r.t & $11 . r$ \\
\hline 4 & $r / r \cdot 1 \cdot-r$ & r.A & M.A & $1 . r$ & $\varepsilon . \wedge$ & r.. & AV.V & r.o & Ir.r \\
\hline 5 & $r / r \cdot 1 \cdot-r$ & $r . \wedge$ & r.l & $1 \ldots$ & ย.V & r.. & AV.T & r.乏 & $11 . v$ \\
\hline 6 & $r / r \cdot 1 .-\Lambda$ & r.V & Tr.I & $9 . r$ & $\varepsilon . \varepsilon$ & ro.1 & AV.T & r.r & 11.2 \\
\hline 7 & $r / r+1 .-1 r$ & T.V & r.r & 9.9 & $\leq .0$ & אד.1 & 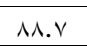 & r.1 & 11.0 \\
\hline 8 & $r / r+1 .-r_{1}$ & r.V & T.t & $9 . \varepsilon$ & $\leq .0$ & ז.. & MA.r & r.t & 11.7 \\
\hline 9 & $r / r+1 .-r v$ & T.V & M.A & 9.9 & $\varepsilon .7$ & r.. & NV. $\varepsilon$ & r.r & $11 . r$ \\
\hline 10 & $0 / 4.1 .-9$ & r.9 & TI. & 9.7 & $\varepsilon . \varepsilon$ & ra.r & 94.0 & r.t & 11.1 \\
\hline 11 & $0 / r+1 .-1 V$ & r.r & M.r & $1 \ldots$ & $\varepsilon .7$ & r9.7 & 9 9r.. & r.乏 & $11 . V$ \\
\hline 12 & $0 / r \cdot 1 .-r V$ & $r .9$ & r. & 1.1 & 纟.V & rᄉ.ی & 19.9 & r.. & Ir.r \\
\hline 13 & $0 / r \cdot 1 .-r_{r}$ & r.V & T. & 9.9 & $\varepsilon .7$ & rᄉ. & 19.0 & r.r & Ir.r \\
\hline 14 & $1 / r \cdot 1 \cdot-1$ & r.A & TI.V & 9.7 & $\varepsilon .0$ & 「ᄉ.T & 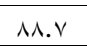 & r.o & IT.r \\
\hline 15 & $r / r \cdot 1 \cdot-r$ & r.9 & Mr.O & $9 . r$ & 纟.० & ru.o & $9 . . r$ & r.o & K. \\
\hline 16 & $7 / Y \cdot 1 .-\varepsilon$ & r.9 & MT.Y & $1 \cdots$ & \&.V & rN.Y & 19.1 & r.乏 & Ir.r \\
\hline 17 & $T / r+1 \cdot-r$ & r.A & rT.I & 9.1 & $\varepsilon . \wedge$ & rv.l & 19.r & r.o & Ir.. \\
\hline 18 & $7 / r \cdot 1 \cdot-9$ & r.. & Tr.t & $9 . r$ & $\varepsilon . \varepsilon$ & rN.r & $9 . . r$ & $r .0$ & IT.Y \\
\hline 19 & $r / r+1 .-1 r$ & r.V & r.r & 9.V & $\varepsilon . \wedge$ & rq.. & qr.r & r.o & Ir.r \\
\hline 20 & $r / r+1,-r \leq$ & r.A & Mr.q & $1 \ldots$ & $\varepsilon .9$ & rᄉ.A & $91 . \varepsilon$ & $r .7$ & Ir.1 \\
\hline 21 & $r / r+1 \cdot-r \Lambda$ & r.. & ק.o & $1 . .7$ & $0 . r$ & rA.V & $9 . .7$ & r.o & Ir.r \\
\hline 22 & $r / r+1 .-r 4$ & r.A & M.O & $9 . r$ & $\leq .0$ & rV. & $9 . . \varepsilon$ & r. & $1 r .7$ \\
\hline 23 & $r / r \cdot 1 .-r_{\Lambda}$ & $r .9$ & Tr.T & $1 \ldots$ & $\leq . \wedge$ & rV.o & 91.0 & r.o & Ir.. \\
\hline 24 & $V / r+1 \cdot-0$ & r.9 & M.A & $1 . r$ & $\leq . \wedge$ & 47.9 & 19.7 & r.乏 & $11 . r$ \\
\hline 25 & $v / r+1 \cdot-q$ & $r .9$ & M.I & $1 . .1$ & $\varepsilon .7$ & ro.v & $\wedge \wedge . \wedge$ & r.r & 11.2 \\
\hline 26 & $V / r+1 .-10$ & r.A & $r \cdot . q$ & $1 \cdot \varepsilon$ & 纟.V & ro.v & AV.V & $r .0$ & Ir.. \\
\hline 27 & $V / r+1 .-I V$ & $r .9$ & r... & $1 \ldots$ & $\varepsilon .0$ & r... & AV. $\varepsilon$ & r.r & IT.r \\
\hline 28 & $9 / r \cdot 1 \cdot-7$ & r. 9 & r.I & $9 . \mathrm{V}$ & $\varepsilon .7$ & 54.9 & $91 . \varepsilon$ & r.乏 & $11 . r$ \\
\hline 29 & $9 / r+1 .-17$ & r... & TI. & 9.1 & $\varepsilon . r$ & r. & $9 . .1$ & r.r & $11 . r$ \\
\hline 30 & $\left.q / r_{0}\right)-r_{0}$ & T.V & $r \cdot . \varepsilon$ & $1 . r$ & $\leq .0$ & rV.r & $9 . . \varepsilon$ & r.乏 & $11 . \varepsilon$ \\
\hline 31 & $\left.9 / r_{0}\right)-r_{0}$ & r.l & M.T & 9.9 & 纟.V & rV. & $9 . . r$ & r.o & $11 . r$ \\
\hline 32 & $q / r+1 .-r$. & r.A & $r . .9$ & 9.0 & $\varepsilon . r$ & rV. & 19.1 & r.t & 11.7 \\
\hline 33 & $9 / r .1 .-r_{0}$ & r.V & (זי. & $9 . \varepsilon$ & $\varepsilon .7$ & אוד & $9 . .9$ & r.r & $11 . \varepsilon$ \\
\hline 34 & $1 r / r \cdot 1 \cdot-11$ & r.V & r.r & 9.1 & 纟.1 & ro.9 & AV.A & r.r & 11.9 \\
\hline 35 & $r r / r \cdot 1 \cdot-r q$ & Y.० & r. & $9 . \varepsilon$ & $\varepsilon . \varepsilon$ & ro.v & 19.. & r.r & $1 Y .1$ \\
\hline 36 & $1 r / r .1 .-r \varepsilon$ & Y.० & $r . .$. & 9.7 & 纟.1 & ro.s & AV. & r.r & Ir.e \\
\hline
\end{tabular}


Table 1 Cont.

\begin{tabular}{|c|c|c|c|c|c|c|c|c|c|}
\hline No. & $\begin{array}{c}\text { Type Plants } \\
\text { No. }\end{array}$ & $\begin{array}{c}\text { Boll } \\
\text { Weight } \\
\text { gm. }\end{array}$ & $\begin{array}{c}\text { Lint } \\
\text { Percent } \\
\quad \%\end{array}$ & $\begin{array}{c}\text { Seed } \\
\text { Index } \\
\text { gm. }\end{array}$ & $\begin{array}{l}\text { Lint } \\
\text { Index } \\
\text { gm. }\end{array}$ & $\begin{array}{c}\text { Fiber } \\
\text { Length } \\
2.5 \% \\
\mathrm{~mm}\end{array}$ & U.R.\% & F.F. & $\begin{array}{c}\text { Pressley } \\
\text { index }\end{array}$ \\
\hline 37 & $I T / \Gamma \cdot 1 \cdot-r V$ & Y.V & Tr.T & 9.. & $\Sigma .0$ & ro.1 & $9 \cdot .5$ & r.乏 & 11.7 \\
\hline 38 & $1 r / \Gamma \cdot \mid \cdot-\Lambda$ & r.l & 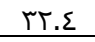 & 9.5 & $\Sigma . \Sigma$ & $r \wedge .1$ & $9 \cdot .0$ & r.乏 & 11.0 \\
\hline 39 & $1 \Gamma / \Gamma \cdot 1 \cdot-17$ & r.•. & rT.I & $9 . \mathrm{V}$ & $\varepsilon .7$ & $r \wedge .0$ & $9 \cdot .1$ & r.o & 11.5 \\
\hline 40 & $\mid r / T \cdot 1 \cdot-1 V$ & r.l & rT.O & $1 \cdot . \Sigma$ & 0. & r^.V & $95 . \cdot$ & r.o & 11.7 \\
\hline 41 & $|r / \Gamma \cdot| \cdot-\Gamma \Lambda$ & Y.Y & r1.9 & 9.9 & $\varepsilon .7$ & 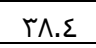 & $9 \cdot .5$ & $r .7$ & $11 . r$ \\
\hline 42 & $10 / \Gamma \cdot 1 \cdot-7$ & $\Gamma . \Lambda$ & rI.V & 9.1 & $\Sigma .0$ & r... & $\Lambda \Lambda . r$ & r.T & $1 T .5$ \\
\hline 43 & $10 / 5 \cdot 1 \cdot-9$ & r.. & ri.. & $9 . \Upsilon$ & $\varepsilon . \Gamma$ & r... & $\Lambda 9 . \vee$ & r.l & $1 T . r$ \\
\hline 44 & $10 / \Gamma \cdot 1 \cdot-r_{0}$ & $r .9$ & 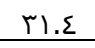 & 9.0 & $\Sigma . r$ & rA.r & 91.1 & T.T & 11.5 \\
\hline 45 & $10 / \Gamma \cdot 1 \cdot-r \Lambda$ & 5.9 & rr.• & 9.7 & $\Sigma . V$ & rV.乏 & $\Lambda 9 . \mathrm{V}$ & r.o & 11.5 \\
\hline 46 & $I V / \Gamma \cdot 1 \cdot-1$ & 5.9 & rI.^ & $9 . \Sigma$ & $\Sigma . \Sigma$ & ro.n & $\Lambda V . \Sigma$ & r.r & $1 T . r$ \\
\hline 47 & $I V / r \cdot 1 \cdot-r T$ & r.r & MT.T & $9 . r$ & $\varepsilon .7$ & ro.9 & $\Lambda V .9$ & r.o & $1 Y . r$ \\
\hline 48 & $I V / \Gamma \cdot \mid \cdot-r \Sigma$ & 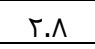 & Mr.P & $1 \cdot . r$ & 0.1 & ro.V & $\Lambda V . V$ & $r .7$ & 11.6 \\
\hline 49 & $1 V / r \cdot 1 \cdot-r q$ & r.r & rr.• & $9 . r$ & $\varepsilon .7$ & ro.r & $\Lambda V .9$ & $r .7$ & $11 . r$ \\
\hline 50 & $r \cdot / r \cdot \mid \cdot-r \Lambda$ & $\Gamma . \Lambda$ & r... & 9.0 & $\varepsilon . \Gamma$ & r.r. & $\Lambda \mathrm{V} . \mathrm{V}$ & r.乏 & 11.5 \\
\hline 51 & $\Gamma \cdot / r \cdot 1 \cdot-\varepsilon \cdot$ & r.l & $r \cdot .1$ & $9 . \Sigma$ & $\Sigma . \cdot$ & ro.9 & $\Lambda 9.5$ & r.s & $11 . r$ \\
\hline 52 & $r \mid / r \cdot 1 \cdot-11$ & r.l & $r \cdot . l$ & $9 . r$ & $\Sigma . \cdot$ & ro. & $\Lambda V . \Sigma$ & r.s & 11.1 \\
\hline 53 & $r_{1} / r \cdot 1 \cdot-r_{0}$ & T.V & rI. & 9.. & $\Sigma . \Gamma$ & ro.9 & $\Lambda \Lambda .0$ & r.乏 & 11.5 \\
\hline 54 & $T \Gamma / T \cdot 1 \cdot-1$ & r.l & $r 1.9$ & $9 . \Sigma$ & $\Sigma . \Sigma$ & r.. & $\Lambda 9.5$ & r.. & $11 . r$ \\
\hline 55 & $r T / \Gamma \cdot 1 \cdot r \Lambda$ & $r .9$ & ri.o & $9 . \mathrm{V}$ & $\Sigma .0$ & r.. 1 & $\Lambda 9.1$ & r.7 & 11.0 \\
\hline 56 & ro/r.1. - 1 . & $\Gamma . \Lambda$ & ri.l & $9 . \Gamma$ & $\Sigma . \Gamma$ & r.. & 91.0 & r.o & $11 . r$ \\
\hline 57 & $r 0 / r \cdot 1 \cdot-r \mid$ & T.V & $r \cdot .7$ & 9.7 & $\Sigma . \Gamma$ & r.. & $\Lambda \Lambda .1$ & $r . \Sigma$ & $11 . \cdot$ \\
\hline 58 & $r V / r \cdot 1 \cdot-r \Sigma$ & 5.9 & ri.o & 10.1 & $\varepsilon .7$ & rV.• & $\Lambda \Lambda . \Lambda$ & $r .7$ & 11.5 \\
\hline 59 & $r V / r \cdot 1 \cdot-r V$ & $\Gamma . \Lambda$ & rI.r & $9 . \mathrm{V}$ & $\Sigma . \Sigma$ & ro.ı & $\Lambda 9.0$ & r.乏 & $11 . \mathrm{V}$ \\
\hline 60 & $r q / r \cdot 1 \cdot-r^{\prime}$ & T.ム & $r 1.9$ & १.V & $\Sigma .0$ & ro.7 & $\Lambda V .7$ & r.r & $\mid T .1$ \\
\hline \multicolumn{2}{|c|}{$\begin{array}{c}\text { Mean of selected type } \\
\text { plants }\end{array}$} & $\ulcorner .87$ & 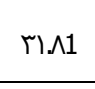 & 9.69 & $\Sigma 52$ & rา.89 & $\wedge 9.41$ & r.37 & 11.70 \\
\hline \multicolumn{2}{|c|}{$\begin{array}{c}\text { Mean of } \\
\text { comparisons (controls) }\end{array}$} & $\ulcorner. \wedge 1$ & $\pi \cdot 2$ & ৭.^1 & $\Sigma V 1$ & ro. 2 & $\Lambda V .71$ & r.o1 & $11 . r 3$ \\
\hline \multicolumn{2}{|c|}{ S. E. } & 0.022 & 0.108 & 0.050 & 0.033 & 0.156 & 0.185 & 0.020 & 0.056 \\
\hline \multicolumn{2}{|r|}{ C. V. $\%$} & 5.91 & 2.62 & 3.98 & 5.59 & 3.27 & 1.61 & 4.59 & 3.73 \\
\hline
\end{tabular}

S. E. $\quad=$ Standard Error.

C. V. $\%=$ Coefficient of variability.

Means of agronomic and fiber properties of the 60 selected progenies (increase A) in 2011 season compared with three latest strains of $G .87$ are given in Table 2. It could be noticed that, the means of progenies (increase A) slightly exceeded the means of comparisons for most traits, except for fiber length $2.5 \%$ in m.m., fiber strength in $\mathrm{g} /$ tex and elongation traits as well as, exhibited by selection better values than the means of comparisons. Coefficients of variability (C.V. \%) were decreased for all the studied characters, indicating gene fixation and homogeneity of variety. 
Table 2. Means of yield components and fiber properties for the 60 selected progenies (increase A) in final of 2011 growing season.

\begin{tabular}{|c|c|c|c|c|c|c|c|c|c|c|c|c|c|}
\hline No. & $\begin{array}{c}\text { Selected } \\
\text { Progenies } \\
\text { No. }\end{array}$ & $\begin{array}{l}\text { B.W. } \\
\text { gm. }\end{array}$ & $\begin{array}{c}\text { L.P. } \\
\%\end{array}$ & $\begin{array}{l}\text { S.I. } \\
\text { gm. }\end{array}$ & $\begin{array}{l}\text { L.I. } \\
\text { gm. }\end{array}$ & $\begin{array}{l}\text { Fib. } \\
\text { Len. } \\
2.5 \% \\
\text { m.m. }\end{array}$ & U.R.\% & $\begin{array}{c}\text { ST. } \\
\text { g./tex }\end{array}$ & F.F. & Elon. & $+b$ & $\begin{array}{l}\text { RD } \\
\%\end{array}$ & $\begin{array}{l}\text { Yarn } \\
\text { Stern. }\end{array}$ \\
\hline 1 & $1 / 2010-5$ & 2.9 & 32.6 & 11.1 & 5.4 & 36.2 & 88.2 & 42.6 & 3.3 & 6.3 & 9.6 & 75.5 & 2990 \\
\hline 2 & $1 / 2010-38$ & 2.9 & 32.5 & 10.3 & 5.0 & 37.4 & 88.3 & 44.2 & 3.4 & 6.6 & 9.4 & 75.2 & 2990 \\
\hline 3 & $1 / r+1 .-r q$ & 3.0 & 33.7 & 11.4 & 5.8 & 36.8 & 88.4 & 47.2 & 3.4 & 6.7 & 9.2 & 74.0 & 3015 \\
\hline 4 & $r / r \cdot 1 \cdot-r$ & 2.8 & 32.3 & 11.5 & 5.5 & 36.6 & 87.8 & 43.2 & 3.4 & 6.7 & 9.2 & 73.6 & 2930 \\
\hline 5 & $r / r \cdot 1 \cdot-r$ & 2.8 & 32.5 & 11.6 & 5.6 & 37.3 & 88.2 & 46.3 & 3.4 & 6.5 & 9.3 & 75.0 & 2940 \\
\hline 6 & $r / r \cdot 1 \cdot-1$ & 2.8 & 33.4 & 11.7 & 5.9 & 37.0 & 87.4 & 46.2 & 3.5 & 6.6 & 9.7 & 74.3 & 2855 \\
\hline 7 & $r / r+1 .-1 r$ & 2.9 & 33.3 & 11.7 & 5.8 & 37.0 & 87.9 & 43.9 & 3.4 & 6.6 & 9.4 & 76.3 & 2900 \\
\hline 8 & $r / r \cdot 1 \cdot-r)$ & 2.8 & 33.1 & 11.3 & 5.6 & 37.8 & 87.9 & 43.0 & 3.2 & 6.2 & 9.2 & 76.4 & 2900 \\
\hline 9 & $r / r+1 .-r v$ & 2.8 & 33.4 & 11.6 & 5.8 & 37.4 & 88.5 & 47.4 & 3.4 & 6.6 & 9.5 & 74.8 & 3030 \\
\hline 10 & $0 / r+1 \cdot-q$ & 3.1 & 33.3 & 11.4 & 5.7 & 37.4 & 88.3 & 46.3 & 3.4 & 6.5 & 9.3 & 75.3 & 2995 \\
\hline 11 & $0 / r+1 .-I V$ & 3.0 & 33.0 & 11.1 & 5.5 & 36.6 & 88.0 & 43.5 & 3.4 & 6.6 & 9.3 & 76.4 & 2910 \\
\hline 12 & $0 / r+1 .-r V$ & 3.1 & 33.6 & 10.8 & 5.5 & 37.0 & 88.1 & 43.7 & 3.6 & 6.6 & 8.8 & 76.6 & 2970 \\
\hline 13 & $0 / r \cdot 1 \cdot-r r$ & 2.9 & 34.3 & 10.9 & 5.7 & 36.5 & 88.7 & 43.5 & 3.5 & 6.3 & 9.0 & 76.4 & 2990 \\
\hline 14 & $r / r \cdot 1 \cdot-1$ & 2.9 & 34.7 & 10.0 & 5.3 & 37.5 & 88.8 & 44.7 & 3.4 & 6.8 & 8.9 & 76.1 & 2960 \\
\hline 15 & $r / r \cdot 1 \cdot-r$ & 3.0 & 34.3 & 10.6 & 5.5 & 37.2 & 88.9 & 43.7 & 3.5 & 6.3 & 8.2 & 75.5 & 2965 \\
\hline 16 & $1 / r \cdot 1 \cdot-\varepsilon$ & 3.1 & 32.4 & 11.5 & 5.5 & 37.4 & 88.5 & 43.1 & 3.5 & 6.7 & 8.8 & 74.4 & 2930 \\
\hline 17 & $T / Y \cdot 1 \cdot-1$ & 3.1 & 32.4 & 11.2 & 5.4 & 37.2 & 88.4 & 42.9 & 3.4 & 6.0 & 9.1 & 74.9 & 2960 \\
\hline 18 & $7 / r+1 \cdot-9$ & 3.1 & 32.7 & 11.3 & 5.5 & 36.0 & 88.4 & 42.7 & 3.5 & 6.0 & 9.4 & 75.5 & 2985 \\
\hline 19 & $r / r \cdot 1 \cdot-1 r$ & 3.1 & 32.6 & 11.3 & 5.5 & 37.3 & 87.4 & 43.8 & 3.8 & 6.6 & 8.8 & 75.8 & 2955 \\
\hline 20 & $T / T \cdot 1 \cdot-r \varepsilon$ & 3.1 & 33.3 & 11.1 & 5.5 & 37.0 & 87.8 & 42.3 & 3.5 & 6.1 & 9.2 & 76.4 & 2910 \\
\hline 21 & $r / r+1 \cdot-r \lambda$ & 3.1 & 33.4 & 11.1 & 5.6 & 36.6 & 87.1 & 42.5 & 3.5 & 6.1 & 8.8 & 76.2 & 2990 \\
\hline 22 & $r / r+1 .-r r$ & 3.1 & 33.5 & 11.5 & 5.8 & 37.4 & 87.6 & 47.2 & 3.4 & 6.7 & 9.0 & 77.5 & 2995 \\
\hline 23 & $r / r+1 .-r \lambda$ & 3.1 & 32.7 & 11.4 & 5.5 & 36.4 & 87.4 & 44.8 & 3.5 & 6.6 & 9.4 & 75.4 & 2980 \\
\hline 24 & $v / r+1 \cdot-0$ & 3.1 & 33.1 & 11.6 & 5.7 & 37.9 & 88.0 & 46.5 & 3.4 & 6.0 & 9.2 & 77.0 & 2870 \\
\hline 25 & $v / r+1 \cdot-q$ & 3.0 & 33.8 & 11.2 & 5.7 & 36.0 & 83.6 & 44.2 & 3.7 & 6.8 & 9.1 & 76.6 & 2995 \\
\hline 26 & $v / r+1 .-10$ & 3.1 & 32.4 & 11.4 & 5.5 & 37.7 & 88.4 & 45.6 & 3.6 & 6.3 & 8.6 & 75.7 & 2900 \\
\hline 27 & $V / r+1 .-I V$ & 2.9 & 33.7 & 10.9 & 5.5 & 36.8 & 87.2 & 41.4 & 3.8 & 6.8 & 8.4 & 77.2 & 2960 \\
\hline 28 & $9 / r \cdot 1 \cdot-7$ & 3.1 & 33.9 & 11.6 & 5.9 & 37.0 & 87.3 & 43.2 & 3.6 & 6.8 & 9.3 & 72.4 & 2970 \\
\hline 29 & $9 / r+1 .-17$ & 3.0 & 34.0 & 10.9 & 5.6 & 36.2 & 88.0 & 43.3 & 3.4 & 6.2 & 9.1 & 75.5 & 2990 \\
\hline 30 & $q / r+1 .-r$. & 3.1 & 34.7 & 10.5 & 5.6 & 36.3 & 88.5 & 42.4 & 3.4 & 6.8 & 8.9 & 77.3 & 2980 \\
\hline 31 & $9 / r_{0} 1 .-r_{0}$ & 3.1 & 33.0 & 11.6 & 5.7 & 37.1 & 88.0 & 45.3 & 3.4 & 6.6 & 8.2 & 77.9 & 2995 \\
\hline 32 & $q / r+1 .-r$. & 3.1 & 35.0 & 11.7 & 6.3 & 36.4 & 88.8 & 40.9 & 3.6 & 6.0 & 9.5 & 74.3 & 2990 \\
\hline 33 & $\left.9 / r_{0}\right) \cdot r_{0}$ & 2.9 & 33.4 & 11.2 & 5.6 & 36.6 & 86.7 & 44.6 & 3.4 & 6.5 & 9.4 & 74.0 & 2910 \\
\hline 34 & $1 r / r \cdot 1 \cdot-11$ & 2.8 & 35.8 & 10.7 & 6.0 & 36.6 & 88.4 & 40.6 & 3.6 & 6.0 & 9.6 & 76.3 & 2980 \\
\hline 35 & $r / r \cdot 1 \cdot-r q$ & 2.9 & 32.9 & 10.6 & 5.2 & 36.7 & 88.5 & 43.7 & 3.6 & 6.7 & 9.2 & 75.5 & 2900 \\
\hline 36 & $r / r \cdot 1 \cdot-r \varepsilon$ & 3.0 & 33.5 & 11.2 & 5.6 & 36.2 & 88.5 & 44.1 & 3.5 & 6.7 & 9.8 & 75.6 & 2910 \\
\hline 37 & $M / r \cdot 1 \cdot-r V$ & 2.9 & 33.9 & 11.3 & 5.8 & 37.1 & 88.8 & 43.7 & 3.4 & 6.5 & 9.6 & 75.0 & 2905 \\
\hline 38 & $1 r / r \cdot 1 \cdot-\lambda$ & 3.1 & 33.3 & 11.1 & 5.5 & 37.0 & 88.1 & 42.9 & 3.6 & 6.2 & 9.8 & 75.5 & 2995 \\
\hline 39 & $1 \pi / r \cdot 1 \cdot-17$ & 3.1 & 32.6 & 11.3 & 5.5 & 37.7 & 88.1 & 43.3 & 3.4 & 6.5 & 9.5 & 75.8 & 2980 \\
\hline 40 & $1 r / r \cdot 1 \cdot-1 r$ & 3.0 & 32.5 & 10.9 & 5.2 & 36.7 & 88.2 & 42.0 & 3.5 & 6.2 & 9.3 & 74.5 & 2950 \\
\hline 41 & $1 r / r \cdot 1 \cdot-r \Lambda$ & 3.0 & 32.3 & 11.3 & 5.4 & 36.3 & 88.6 & 44.4 & 3.5 & 6.6 & 9.4 & 75.7 & 3115 \\
\hline
\end{tabular}


Table 2 Cont.

\begin{tabular}{|c|c|c|c|c|c|c|c|c|c|c|c|c|c|}
\hline No. & $\begin{array}{c}\text { Selected } \\
\text { Progenies } \\
\text { No. }\end{array}$ & $\begin{array}{l}\text { B.W. } \\
\text { gm. }\end{array}$ & $\begin{array}{c}\text { L.P. } \\
\%\end{array}$ & $\begin{array}{l}\text { S.I. } \\
\text { gm. }\end{array}$ & $\begin{array}{l}\text { L.I. } \\
\text { gm. }\end{array}$ & $\begin{array}{l}\text { Fib. Len. } \\
2.5 \% \\
\text { m.m. }\end{array}$ & U.R.\% & $\begin{array}{c}\text { ST. } \\
\text { g./tex }\end{array}$ & F.F. & Elon. & $+b$ & RD \% & $\begin{array}{c}\text { Yarn } \\
\text { Stern. }\end{array}$ \\
\hline 42 & $10 / r+1 .-7$ & 2.9 & 34.7 & 11.1 & 5.9 & 37.0 & 88.7 & 42.2 & 3.5 & 6.4 & 9.8 & 74.9 & 2965 \\
\hline 43 & $10 / r \cdot 1 .-9$ & 3.0 & 33.7 & 11.5 & 5.8 & 37.0 & 87.8 & 46.0 & 3.5 & 6.5 & 9.0 & 74.5 & 2995 \\
\hline 44 & $10 /$ r.1. - ro & 2.9 & 32.3 & 10.6 & 5.1 & 37.1 & 87.7 & 45.8 & 3.6 & 6.4 & 9.4 & 72.7 & 2910 \\
\hline 45 & $10 / r \cdot 1 .-r_{1}$ & 3.0 & 33.6 & 11.0 & 5.6 & 37.2 & 88.1 & 42.3 & 3.5 & 6.3 & 9.8 & 75.5 & 2960 \\
\hline 46 & $I V / r \cdot 1 \cdot-1$ & 2.9 & 32.8 & 11.1 & 5.4 & 36.3 & 88.4 & 45.1 & 3.7 & 6.1 & 9.0 & 75.1 & 2980 \\
\hline 47 & $I V / r \cdot I \cdot-r r$ & 3.2 & 33.5 & 11.1 & 5.6 & 36.4 & 88.2 & 42.4 & 3.5 & 6.3 & 8.6 & 73.8 & 2760 \\
\hline 48 & $I V / r \cdot 1 \cdot-r \varepsilon$ & 3.0 & 33.6 & 10.8 & 5.5 & 37.6 & 88.6 & 43.2 & 3.5 & 6.6 & 9.1 & 76.5 & 2825 \\
\hline 49 & $r v / r \cdot 1 \cdot-r q$ & 2.9 & 32.6 & 10.9 & 5.3 & 36.8 & 88.8 & 44.6 & 3.4 & 6.6 & 9.0 & 73.1 & 2865 \\
\hline 50 & $r \cdot / r \cdot l \cdot-r_{\Lambda}$ & 3.0 & 33.5 & 11.2 & 5.6 & 37.1 & 88.2 & 41.2 & 3.5 & 6.2 & 8.7 & 74.6 & 2935 \\
\hline 51 & $\left.r \cdot / r_{\cdot}\right) \cdot-\varepsilon \cdot$ & 2.8 & 33.7 & 11.0 & 5.6 & 36.0 & 87.2 & 40.1 & 3.4 & 6.0 & 8.7 & 77.0 & 2950 \\
\hline 52 & $r / r \cdot 1 \cdot-11$ & 3.1 & 32.8 & 11.3 & 5.5 & 37.1 & 87.8 & 43.2 & 3.5 & 6.2 & 8.9 & 73.8 & 2970 \\
\hline 53 & $r_{1} / r_{0} \cdot{ }^{\prime}-r_{0}$ & 3.1 & 33.7 & 11.3 & 5.7 & 37.9 & 87.3 & 42.2 & 3.3 & 6.3 & 8.6 & 71.3 & 2950 \\
\hline 54 & $r r / r \cdot 1 \cdot-1$ & 3.1 & 34.1 & 10.9 & 5.6 & 35.8 & 88.7 & 40.2 & 3.6 & 6.0 & 8.8 & 72.6 & 2920 \\
\hline 55 & $r r / r \cdot 1 \cdot-r_{\Lambda}$ & 3.0 & 34.1 & 10.5 & 5.4 & 36.5 & 86.0 & 43.8 & 3.4 & 6.5 & 9.1 & 74.5 & 2945 \\
\hline 57 & $\left.r_{0} / r_{0}\right) \cdot r_{1}$ & 2.9 & 33.3 & 10.6 & 5.3 & 37.8 & 88.4 & 45.2 & 3.4 & 6.6 & 9.2 & 75.1 & 2915 \\
\hline 58 & $r v / r \cdot 1 \cdot-r \varepsilon$ & 2.9 & 32.3 & 11.3 & 5.4 & 36.9 & 88.8 & 43.9 & 3.5 & 6.5 & 9.1 & 75.6 & 2950 \\
\hline 59 & $r V / r \cdot 1 \cdot-r r$ & 3.1 & 33.1 & 11.2 & 5.5 & 37.4 & 88.4 & 40.4 & 3.6 & 6.3 & 9.1 & 73.2 & 2890 \\
\hline 60 & $r q / r \cdot 1 \cdot-r \cdot$ & 2.9 & 33.9 & 10.4 & 5.3 & 37.7 & 88.7 & 42.7 & 3.5 & 6.4 & 9.5 & 74.6 & 2760 \\
\hline \multicolumn{2}{|c|}{ Mean of selected progenies } & 2.99 & 33.36 & 11.12 & 5.56 & 36.95 & 88.04 & 43.63 & 3.49 & 6.42 & 9.16 & 75.20 & 2950 \\
\hline \multicolumn{2}{|c|}{ Mean of comparisons (controls) } & 2.91 & 33.02 & 11.10 & 5.52 & 37.10 & 87.81 & 44.01 & 3.51 & 6.50 & 9.30 & 75.19 & 2930 \\
\hline \multicolumn{2}{|c|}{ S. E. } & 0.014 & 0.097 & 0.049 & 0.028 & 0.072 & 0.106 & 0.225 & 0.014 & 0.032 & 0.049 & 0.172 & 7.732 \\
\hline \multicolumn{2}{|c|}{ C. v. $\%$} & 3.65 & 2.25 & 3.41 & 3.96 & 1.50 & 0.94 & 4.00 & 3.21 & 3.86 & 4.14 & 1.78 & 2.03 \\
\hline
\end{tabular}

S. E. = Standard Error.

C. V. $\%=$ Coefficient of variability. 
Table 3. Means of yield, yield components and fiber properties of the 17 selected families (increase B) in 2012 season, furnishing nucleolus in 2013.

\begin{tabular}{|c|c|c|c|c|c|c|c|c|c|c|c|c|c|c|c|c|}
\hline No. & \multicolumn{2}{|c|}{$\begin{array}{l}\text { Selected } \\
\text { Families }\end{array}$} & $\begin{array}{l}\text { S.C.Y. } \\
\text { K./F. }\end{array}$ & L.C.Y. & $\begin{array}{l}\text { B.W. } \\
\text { gm. }\end{array}$ & L.P. & $\begin{array}{l}\text { S.I. } \\
\text { gm. }\end{array}$ & $\begin{array}{l}\text { L.I. } \\
\text { gm. }\end{array}$ & $\begin{array}{c}\text { Fib. Len. } \\
2.5 \% \\
\text { m.m. }\end{array}$ & U.R.\% & ST. & Mic. & Elon. & $+b$ & $\begin{array}{l}\text { RD } \\
\%\end{array}$ & $\begin{array}{l}\text { Yarn } \\
\text { stern. }\end{array}$ \\
\hline 1 & \multicolumn{2}{|c|}{$1 / r \cdot 1 \cdot-r \Lambda$} & 5.8 & 5.5 & 2.9 & 33.2 & 11.2 & 5.6 & 35.5 & 88.3 & 45.0 & 7.5 & 3.3 & 72.5 & 8.8 & 3145 \\
\hline 2 & \multicolumn{2}{|c|}{$1 / r \cdot 1 \cdot-r q$} & 6.9 & 6.9 & 3.0 & 31.7 & 11.1 & 5.1 & 34.5 & 87.0 & 43.4 & 7.2 & 3.3 & 72.4 & 8.7 & 2970 \\
\hline 3 & \multicolumn{2}{|c|}{$r / r \cdot 1 \cdot-r$} & 5.9 & 5.5 & 2.9 & 33.7 & 11.3 & 5.8 & 35.5 & 86.1 & 43.0 & 7.5 & 3.3 & 70.5 & 8.3 & 2960 \\
\hline 4 & \multicolumn{2}{|c|}{$r / r \cdot 1 \cdot-r v$} & 5.4 & 5.4 & 3.0 & 32.2 & 11.4 & 5.4 & 34.6 & 86.8 & 43.1 & 7.3 & 3.3 & 74.5 & 9.9 & 2975 \\
\hline 5 & \multicolumn{2}{|c|}{$0 / r \cdot 1 .-9$} & 4.9 & 4.8 & 2.8 & 31.9 & 11.1 & 5.2 & 34.9 & 86.4 & 42.3 & 7.3 & 3.3 & 74.2 & 8.5 & 2970 \\
\hline 6 & \multicolumn{2}{|c|}{$0 / Y \cdot 1 \cdot-r V$} & 7.1 & 6.8 & 2.9 & 33.1 & 11.0 & 5.5 & 35.2 & 88.9 & 45.7 & 7.4 & 3.3 & 74.1 & 8.6 & 3165 \\
\hline 7 & \multicolumn{2}{|c|}{$7 / Y \cdot 1 .-1$} & 6.9 & 6.7 & 2.9 & 32.8 & 11.0 & 5.4 & 35.7 & 88.8 & 45.4 & 7.2 & 3.3 & 72.7 & 8.2 & 3180 \\
\hline 8 & \multicolumn{2}{|c|}{$r / r \cdot 1 \cdot-r r$} & 6.1 & 6.1 & 2.9 & 31.7 & 11.3 & 5.2 & 35.7 & 89.4 & 46.8 & 7.5 & 3.3 & 75.6 & 8.7 & 3130 \\
\hline 9 & \multicolumn{2}{|c|}{$v / r . l \cdot-0$} & 6.8 & 6.9 & 2.9 & 31.3 & 11.2 & 5.1 & 35.4 & 88.9 & 45.4 & 7.5 & 3.3 & 72.9 & 8.6 & 3120 \\
\hline 10 & \multicolumn{2}{|c|}{$v / r+1 \cdot-10$} & 7.0 & 6.8 & 2.9 & 32.7 & 11.2 & 5.4 & 35.0 & 86.7 & 45.8 & 7.5 & 3.3 & 73.1 & 8.8 & 3130 \\
\hline 11 & \multicolumn{2}{|c|}{$q / r \cdot 1 \cdot-r_{0}$} & 5.7 & 5.6 & 2.9 & 31.9 & 11.2 & 5.3 & 35.3 & 87.2 & 45.3 & 7.1 & 3.1 & 72.9 & 9.6 & 3165 \\
\hline 12 & \multicolumn{2}{|c|}{$1 r / r \cdot 1 \cdot-17$} & 6.6 & 6.6 & 3.0 & 31.5 & 11.3 & 5.2 & 36.8 & 87.7 & 42.6 & 7.0 & 3.1 & 72.9 & 9.0 & 2970 \\
\hline 13 & \multicolumn{2}{|c|}{$10 / r \cdot 1 \cdot-9$} & 6.6 & 6.4 & 3.0 & 32.4 & 11.4 & 5.5 & 32.5 & 85.7 & 43.5 & 7.3 & 3.1 & 77.5 & 9.0 & 2945 \\
\hline 14 & \multicolumn{2}{|c|}{$10 /$ r.l. - ro } & 5.1 & 5.1 & 2.8 & 31.9 & 11.2 & 5.2 & 35.5 & 88.3 & 44.0 & 7.0 & 3.3 & 72.3 & 9.6 & 3090 \\
\hline 15 & \multicolumn{2}{|c|}{$r r / r \cdot l \cdot-r \Lambda$} & 5.3 & 5.3 & 2.9 & 32.1 & 11.0 & 5.2 & 35.0 & 88.3 & 46.3 & 7.5 & 3.3 & 72.4 & 9.4 & 3100 \\
\hline 16 & \multicolumn{2}{|c|}{ ro/r.l. $-r_{1}$} & 6.0 & 5.8 & 2.9 & 32.9 & 11.1 & 5.4 & 34.3 & 85.3 & 45.9 & 7.5 & 3.3 & 72.3 & 9.2 & 2955 \\
\hline 17 & \multicolumn{2}{|c|}{$r V / r \cdot 1,-r \varepsilon$} & 6.0 & 6.0 & 3.0 & 32.0 & 11.3 & 5.3 & 34.0 & 86.9 & 45.2 & 7.5 & 3.3 & 74.5 & 9.0 & 3000 \\
\hline \multicolumn{3}{|c|}{$\begin{array}{c}\text { Mean of selected } \\
\text { Families }\end{array}$} & 6.12 & 6.01 & 2.92 & 32.29 & 11.19 & 534 & 35.02 & 87.45 & 44.63 & 7.34 & 3.26 & 73.37 & 8.94 & 3057 \\
\hline \multicolumn{3}{|c|}{ C.V. $\%$} & 1151 & 1155 & 2.18 & 2.07 & 1.16 & 3.51 & 2.61 & 1.41 & 3.14 & 2.51 & 2.41 & 2.16 & 5.32 & 2.93 \\
\hline \multicolumn{3}{|c|}{$\begin{array}{c}\text { Mean of } \\
\text { comparisons(controls) }\end{array}$} & 6.81 & 6.72 & 2.99 & 3202 & 10.91 & 5.20 & 35.00 & 86.81 & 43.13 & 7.13 & 3.40 & 72.71 & 9.30 & 3045 \\
\hline \multirow{2}{*}{\multicolumn{3}{|c|}{ L.S.D. }} & N.S & N.S & N.S & 1.149 & N.S & 0.358 & \multirow{2}{*}{--} & \multirow{2}{*}{--} & \multirow{2}{*}{--} & \multirow{2}{*}{--} & \multirow{2}{*}{--} & \multirow{2}{*}{--} & \multirow{2}{*}{--} & 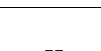 \\
\hline & & & N.S & N.S & N.S & 1.529 & N.S & 0.409 & & & & & & & & -- \\
\hline
\end{tabular}

N.S: Non Significant.
C. V. $\%=$ Coefficient of variability. 
Table 3 shows, the results of the means of yield, yield components and fiber properties for 17 selected families (increase B) compared with the latest strains of G.87 (controls). The results showed that no significant differences were detected among the families and controls for all the studied characters except for lint percentage and lint index. The results are in agreement with those obtained by AbdelAl(1976), Abo-Arab et al., (1995), Lasheen (1997), El-Disoqui (2001), Nagib and Hemida (2001) , Abd Al-Zaher (2004) and Mohamed (2013).

Regarding the results of the 6 selected families Table 4 were not significant compared with the control in yield and other agronomic characters, fiber properties as well as seed quality. Pure seeds of the 6 selected families as the last step in such maintaining program, were massed together to form the breeders seed stock of Giza 87 variety in 2013 season, under name (Giza 87 nucleolus /2013).Table 4 presented the characters of the selected families. 
Table 4. Means of studied characters of 6 families selected from increase B in 2012 growing season to form new nucleolus (breeder's seed) of G. 87 in 2013 season.

\begin{tabular}{|c|c|c|c|c|c|c|c|c|c|c|c|c|c|c|}
\hline $\begin{array}{l}\text { Selected } \\
\text { families }\end{array}$ & $\begin{array}{l}\text { S.C.Y. } \\
\text { K./F. }\end{array}$ & $\begin{array}{l}\text { L.C.Y. } \\
\text { K./F. }\end{array}$ & $\begin{array}{l}\text { B.W. } \\
\text { gm. }\end{array}$ & $\begin{array}{l}\text { L.P. } \\
\%\end{array}$ & $\begin{array}{l}\text { S.I. } \\
\text { gm. }\end{array}$ & $\begin{array}{l}\text { L.I. } \\
\text { gm. }\end{array}$ & $\begin{array}{c}\text { Fib. Len. } \\
2.5 \% \\
\text { m.m. }\end{array}$ & U.R.\% & $\begin{array}{c}\text { ST. } \\
\text { g./tex }\end{array}$ & Mic. & Elon. & $+b$ & RD \% & $\begin{array}{l}\text { Yarn } \\
\text { stern. }\end{array}$ \\
\hline $1 / \Gamma \cdot I \cdot-r \Lambda$ & 5.5 & 5.8 & 2.9 & 33.2 & 11.2 & 5.6 & 35.5 & 88.3 & 45.0 & 3.3 & 7.5 & 8.8 & 72.5 & 3145 \\
\hline$p / r \cdot 1 \cdot-r V$ & 6.8 & 7.1 & 2.9 & 33.1 & 11.0 & 5.5 & 35.2 & 88.9 & 45.7 & 3.3 & 7.4 & 8.6 & 74.1 & 3165 \\
\hline$y / r \cdot 1 \cdot-1$ & 6.7 & 6.9 & 2.9 & 32.8 & 11.0 & 5.4 & 35.7 & 88.8 & 45.4 & 3.3 & 7.2 & 8.2 & 72.7 & 3180 \\
\hline $7 / \Gamma \cdot 1 \cdot-r 7$ & 6.1 & 6.1 & 2.9 & 31.7 & 11.3 & 5.2 & 35.7 & 89.4 & 46.8 & 3.3 & 7.5 & 8.7 & 75.6 & 3130 \\
\hline$N / T \cdot 1 \cdot-0$ & 6.8 & 6.9 & 2.9 & 31.3 & 11.2 & 5.1 & 35.4 & 88.9 & 45.4 & 3.3 & 7.5 & 8.6 & 72.9 & 3120 \\
\hline$N / T \cdot 1 \cdot-10$ & 6.8 & 7.0 & 2.9 & 32.7 & 11.2 & 5.4 & 35.0 & 86.7 & 45.8 & 3.3 & 7.5 & 8.8 & 73.1 & 3130 \\
\hline $\begin{array}{c}\text { Mean of selected } \\
\text { Families }\end{array}$ & 6.5 & 6.6 & T.9 & YT.O & 11.5 & $0 . \Sigma$ & 35.4 & 88.5 & 45.7 & 3.3 & 7.4 & 8.6 & 73.5 & 3145 \\
\hline $\begin{array}{c}\text { Mean of } \\
\text { comparisons(controls) }\end{array}$ & 7.V & $7 . \Lambda$ & r.• & YT.• & $1 \cdot .9$ & $0 . \Gamma$ & 35.3 & 86.8 & 43.1 & 3.4 & 7.1 & 9.3 & 72.7 & 3045 \\
\hline C.V. \% & 8.36 & 8.18 & 0.00 & 2.41 & 1.10 & 3.47 & 0.79 & 1.07 & 1.35 & 0.00 & 1.63 & 2.59 & 1.60 & 0.74 \\
\hline
\end{tabular}

Yield per feddan was calculated from the mean plot size.

C. V. $\%=$ Coefficient of variability. 
The breeder's seed (nucleolus) was grown in 2013 season in 15 feddans at Sakha Farm.

These results provide good evidence that the pure seed stock released by the cotton breeder would be maintained pure as the stocks and exclusively remained under the upper hand of the breeder. Being then the breeder's seed (nucleolus) is further increased to produce the foundation seed as a new cultivar strain carrying the number of the year of its propagation.

On the other hand, deterioration may be occur in cotton varieties in general cultivation through, contamination by mechanical mixing of seeds, out crossing with inferior, foreign cultivars, and off-types which could result in a genetic change of the variety. The results are in agreement with those obtained by Abdel-Bary and Bisher (1969), Abdel-Al (1976), El-Akkad et. al. (1982), El-kilany and Youssef (1985), Younis et. al. (1993), Abo-Arab et. al. (1995), Lasheen (1997), El-Disoqui (2001), Nagib and Hemida (2001), Abd Al-Zaher (2004) and Mohamed (2013).

The pure line method in the sense of pedigree selection for renewing Giza 87 breeder's seed depends on independent culling selection for most characters, this means that the selection technique for producing breeder seed of Giza 87 variety was valid and proved to be effective in holding this variety according to the standard type of Giza 87.

Finally Cotton Varietal Maintainance Research Department, is responsible to produce of nucleolus annually by pedigree selection method as conventional breeding procedure, for maintain the variety on high genetic purity seeds and matching the specification and important characteristics of the variety.

\section{REFERENCES}

1. Abdel-Al, M.S.M. 1976. Some aspects of breeding method for maintaining Egyptian cotton varieties. Ph.D. Thesis Al-Azhar Univ.

2. Abdel-Bary, A.A. and M.A. Bishr. 1969. Evaluation of the new cotton variety Giza 69. Cott. Gr. Rev., 46: 98-104.

3. Abd Al-Zaher, G.H. 2004. Maintenance and producing the nucleolus (breeder's seed) of Giza 83 Egyptian cotton variety, during 2000-2004 seasons. Egypt. J. plant Breeding 8: 77-86.

4. Abo-Arab A.R., A.E. Ayoub and A.F. Lasheen. 1995. Maintenance and producing the nucleolus (breeder's seed) of Giza 76 Egyptian cotton variety, during 19901992 seasons. Zagazig J. Agric. Res., 22 (2): 399-408. 
5. Al-Didi, M.A. 1974. Methods of cotton breeding. Egypt. Cott. Giza, 62: 49-92.

6. El-Akkad M.H.; A.F.H. El-Okkia; H.R. El-Hanafi and M.A. Abdel-Dayem. 1982. Plan for maintenance and producing the nucleolus (breeder's seed) of "Giza 69" Egyptian cotton variety, during 1975-1979 seasons. Agic. Res. Rev., Vol. 60 No. 9 (1982): 111-131.

7. El-Disouqi, A.E. 2001. Maintenance system of Giza 70 Egyptian cotton cultivar .J.Agric. Sci. Mansoura Univ., 26 (4):1853 - 1862.

8. El-Kilany M.A. and S.M. Youssef. 1985. Comparative study on six nuclei seeds of Dendera cotton cultivar and the corresponding farmer's seed in general use. Agric. Res .Rev., Vol. 63 No. 6 (1985) : 53 -59.

9. Lasheen A.F. 1997. Maintenance and producing the nucleolus (breeder's seed) of Giza 75 Egyptian cotton variety. Menofiya J. Agric. Res., 22 (5): 1279-1290.

10. Lewis, C.F. 1970. Concepts of varietal maintenance in cotton. Cott. Gr. Rev., 47: $272-284$.

11. Mohamed, A.A. 2013. Maintenance and producing the nucleolus (breeder's seed) of Giza 90 Egyptian cotton variety, during 2009-2012 seasons. J. Agric. Res. Kafr El-Sheikh Univ., Vol. (39), No. (1), 2013: 79-91.

12. Nagib, M.A.A. and G.M. Hemida. 2001. Some aspects on cotton variety renewal and maintenance scheme of Giza 80. Minia J. Of Agric. Res. of Devolop. 21 (1): $67-75$.

13. Riggs, T. J. 1967. Response to model selection in Upland cotton in northern and eastern Uganda. Cott. Gr. Rev., 44: 176 - 183.

14. Turner, J.H. 1963. Breeding methods used in maintenance and improvement of Acala 4-42 variety of cotton. U.S. Dept. Ar. ARS Cotton Res. Sta. Shafter. Calif., $34-51$.

15. Walker, J.T. 1964. Model Selection in Upland Cotton. Heredity, 19: 559 - 583.

16. Ware, J.O. 1959. Plan for breeding, maintenance and propagation of Egyptian cotton varieties. A report submitted to the Ministry of Agri., Egypt.

17. Younis F.G., E.M. Ghoneim and M.O. Ismail. 1993. Producing the nucleolus (breeder's seed) of "Dendera" Egyptian cotton variety, during 1988 -1991 seasons. Egypt. Jape. Sci., 8 (2): $238-248$. 
خطة التربية والمحافظة و إنتاج النوية (بذرة المربى) لصنف القطن المصري جيزة

محمد عبد المولى الأمير

$$
\text { معهد بحوث القطن - مركز البحوث الزراعية - الجيزة - مصر }
$$

يوضح هذا البحث إنتاج بذرة المربى في برنامج المحافظة على الصنف جيزة NV وهو مسن

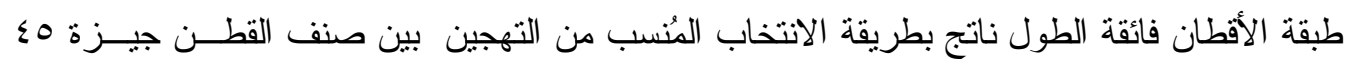

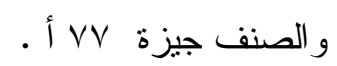

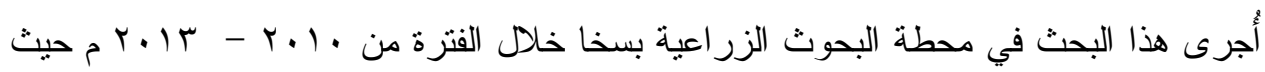

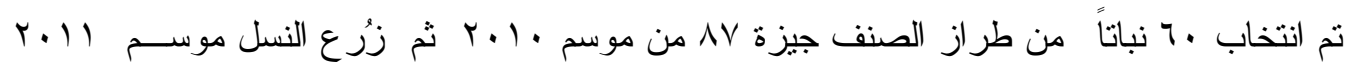

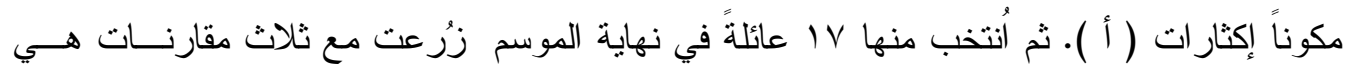

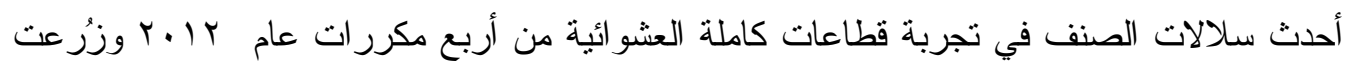
بذرتها الذاتي في مساحة مجاورة للتجربة.

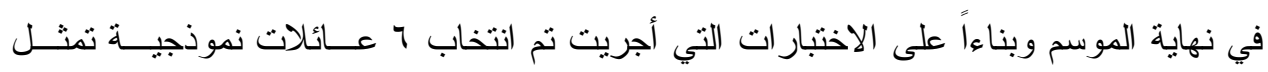

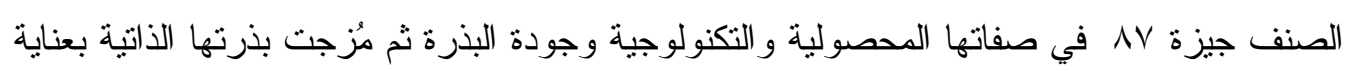

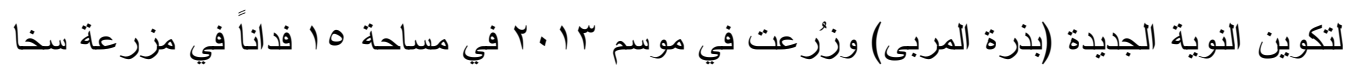

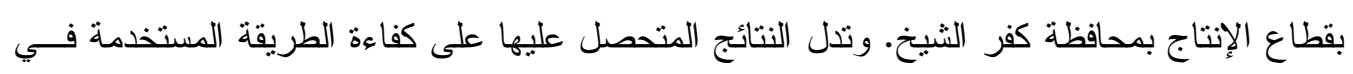
المحافظة على النقاوة الور اثية للصنف جيزة ^V وذللك باستعمال مزج بذرة الأنسال المنتخبة.

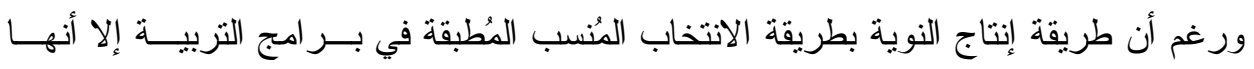

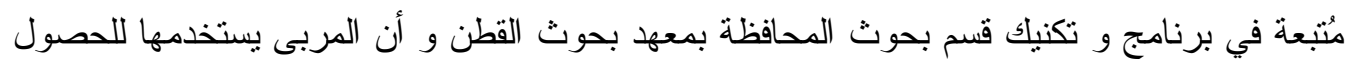

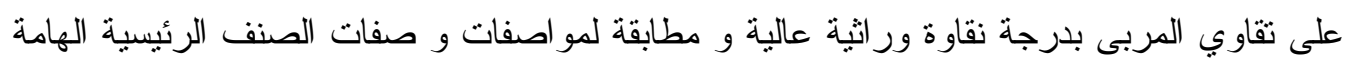

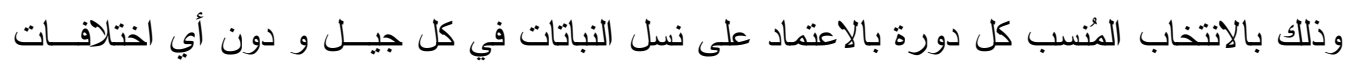

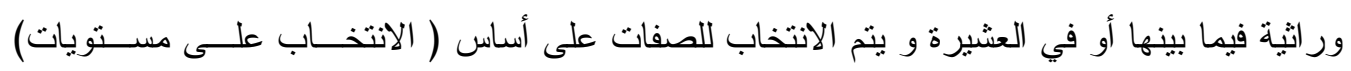

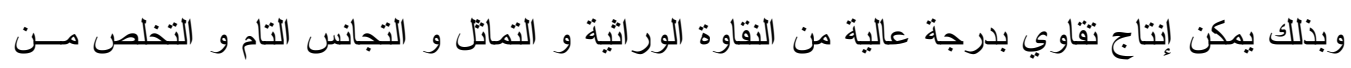
التأثثر الضار من الظروف البيئية و ملاحظة أب اختلافات و استبعادها وذلك لأن الأصناف المصرية

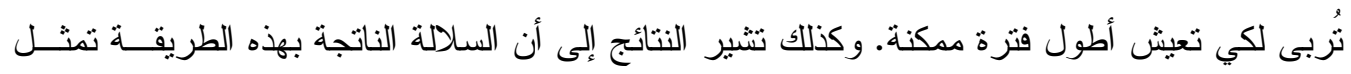

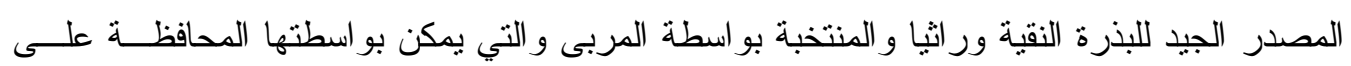
النقاوة الور انية للصنف جيزة Av. 